\title{
Endoscope-assisted repair of CSF otorrhea and temporal lobe encephaloceles via keyhole craniotomy
}

\author{
Pamela C. Roehm, MD, PhD, ${ }^{1-3}$ Derrick Tint, MD, ${ }^{1}$ Norman Chan, MD, ${ }^{1}$ Ryan Brewster, BA, ${ }^{4}$ \\ Vishad Sukul, MD, ${ }^{5}$ and Kadir Erkmen, MD ${ }^{3}$

\begin{abstract}
Departments of ${ }^{1}$ Otolaryngology, ${ }^{2}$ Neuroscience, and ${ }^{3}$ Neurosurgery, Temple University School of Medicine, Philadelphia, Pennsylvania; ${ }^{4}$ Department of Pathology, Brigham and Women's Hospital, Boston, Massachusetts; and ${ }^{5}$ Department of
\end{abstract} \\ Neurosurgery, Vanderbilt University Medical Center, Nashville, Tennessee
}

\begin{abstract}
OBJECTIVE Temporal lobe encephaloceles and cerebrospinal fluid otorrhea from temporal bone defects that involve the tegmen tympani and mastoideum are generally repaired using middle fossa craniotomy, mastoidectomy, or combined approaches. Standard middle fossa craniotomy exposes patients to dural retraction, which can lead to postoperative neurological complications. Endoscopic and minimally invasive techniques have been used in other surgeries to minimize brain retraction, and so these methods were applied to repair the lateral skull base. The goal of this study was to determine if the use of endoscopic visualization through a middle fossa keyhole craniotomy could effectively repair tegmen defects.
\end{abstract}

METHODS The authors conducted a retrospective review of 6 cases of endoscope-assisted middle fossa repairs of tegmen dehiscences at a tertiary care medical center within an 18-month period.

RESULTS All cases were successfully treated using a keyhole craniotomy with endoscopic visualization and minimal retraction. Surgical times did not increase. There were no major postoperative complications, recurrences of encephaloceles, or cerebrospinal fluid otorrhea in these patients.

CONCLUSIONS Endoscopic visualization allows for smaller incisions and craniotomies and less risk of brain retraction injury without compromising repair integrity during temporal encephalocele and tegmen repairs.

https://thejns.org/doi/abs/10.3171/2017.1.JNS161947

KEY WORDS cerebrospinal fluid otorrhea; endoscope-assisted; endoscopic skull base surgery; keyhole craniotomy; middle fossa craniotomy; temporal lobe encephalocele; surgical technique

$\mathrm{C}$ EREBROSPINAL fluid (CSF) otorrhea and temporal lobe encephaloceles (TLEs) involving the tegmen tympani and mastoideum are relatively rare neurotologic conditions that have become more common in the past 10 years..$^{10}$ Increases in the incidence of these conditions are linked to the trend toward higher body weight and sleep apnea in the US population.,10,14 These conditions result from defects in the tegmen tympani and/or mastoideum. Cerebrospinal fluid otorrhea and TLE are associated with serious complications such as meningitis and brain abscesses, as well as conductive hearing loss and chronic middle ear effusion. ${ }^{11}$

Appropriate management of TLEs and chronic CSF otorrhea requires surgical repair through a middle fossa craniotomy, transmastoid approach, or combined approach. ${ }^{5,8,11,14}$ Spontaneous TLE or CSF otorrhea can occur from multiple dehiscences in the tegmen, which typically makes repair via middle fossa craniotomy more successful. However, middle fossa craniotomy exposes patients to extradural retraction of the temporal and parietal cortices and the use of dehydrating agents to allow exposure, which can lead to temporary deficits in cognitive function and an increased risk of cerebrovascular accident. ${ }^{1-3,7,12}$ In this report we describe endoscope-assisted middle fossa craniotomy to repair TLEs and CSF otorrhea due to tegmen dehiscence. The use of endoscopes for visualization of the tegmen allows for a smaller skin incision, a small craniotomy, and less retraction on the temporal lobe than with

ABBREVIATIONS BMI = body mass index; CSF = cerebrospinal fluid; PTA = pure tone average; TLE = temporal lobe encephalocele.

SUBMITTED July 26, 2016. ACCEPTED January 26, 2017.

INCLUDE WHEN CITING Published online August 11, 2017; DOI: 10.3171/2017.1.JNS161947. 
a traditional middle fossa craniotomy approach. Here we describe the technique and a series of 5 patients who have undergone this procedure.

\section{Methods}

This study was approved by the institutional review board of our medical school. Given its retrospective nature and low risk for patients, the study was granted exempt status.

The operative reports and postoperative records of 5 patients who had undergone 6 endoscope-assisted repairs of the tegmen mastoideum or tegmen tympani through a keyhole craniotomy in an 18-month period were reviewed. Demographic data including age at surgery, sex, and body mass index (BMI) were recorded and analyzed. Details of the surgical procedure and intraoperative measurements of CSF pressure were documented and compared between patients. Details of the postoperative course including total length of hospital stay, length of intensive care unit stay, and duration of lumbar drainage were collected. Preoperative and postoperative audiographic data, including 4-tone pure tone average (PTA; 500, 1000, 2000, and $3000 \mathrm{~Hz}$ ), air-bone gaps, word recognition scores, and results of tympanography were recorded. The data were then stripped of all HIPAA-protected information (e.g., patient name, date of birth, hospital number).

\section{Surgical Technique}

The patient is brought into the operating room and orotracheally intubated by the anesthesia staff. A lumbar drain is placed with the patient in the lateral position. The patient is then placed supine. Facial nerve monitoring and brainstem auditory evoked responses (BAERs) are measured intraoperatively.

Because of the small size of the incision, minimal hair shaving is required. A $4.5-\mathrm{cm}$ lazy-S incision is made through the skin, temporalis fascia, and temporalis muscle above the pinna of the affected ear, with the anterior limb hidden in the preauricular crease (Fig. 1A). A burr hole is then made through the calvaria over the zygomatic ridge. All bone pate and bone dust generated are saved on the back table for later use. The burr hole is then expanded 0.5 $\mathrm{cm}$ anteriorly using a Kerrison rongeur.

All surgeries are performed with 2 operating surgeons. During endoscopic visualization, one surgeon holds the endoscope and suction while the other performs a 2-handed dissection and repair of the defect.

For endoscope-assisted cases, the operating microscope is advanced into the field to provide visualization during elevation of the dura mater from the floor of the middle fossa. For completely endoscopic surgeries, the $0^{\circ}$ nasal endoscope would be used for visualization during this portion of the procedure. The sites of tegmen dehiscence and encephalocele are then identified using a $0^{\circ}, 30^{\circ}$, or $70^{\circ}$ nasal endoscope, depending on the inclination angle of the tegmen at the site of dehiscence (Fig. 2). An endoscopic lens-cleaning sheath (Endo-Scrub 2, Medtronic) is used to facilitate visualization. If a TLE is present, it is either reduced back into position or truncated with bipolar cautery. Dural defects are repaired by overlying the affect-
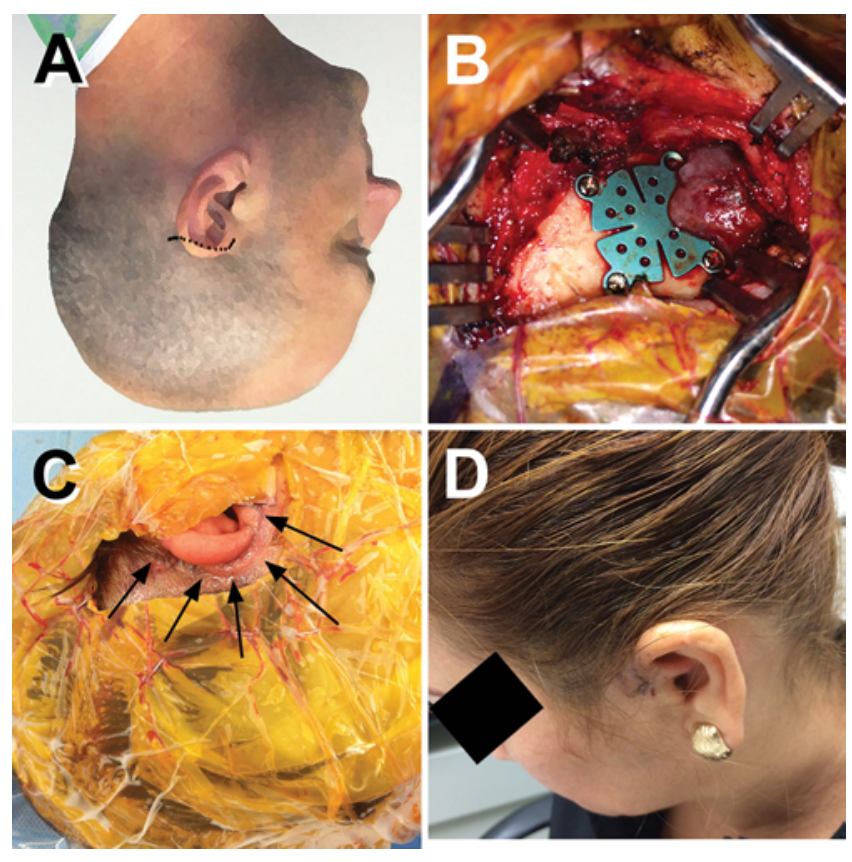

FIG. 1. The lazy-S incision (black dotted and solid line) used for endoscope-assisted middle fossa keyhole craniotomy tegmen repairs has minimal cosmetic impact (A). An extraventricular drain burr hole cover is used for cranioplasty (B). Intraoperative view of closed incision (C); arrows point to incision line. The lazy-S incision 1 week following surgery (D). Figure is available in color online only.

ed areas with a collagen matrix dural substitute (Duragen, Integra LifeSciences Corp.). Pieces of calvarial bone from the burr hole are used to cover dehiscences in the tegmen, and then these areas are coated with bone pate and fibrin sealant (Tisseel, Baxter Healthcare Corp.). A $5 \times 1-\mathrm{cm}$ anteriorly pedicled temporalis muscle flap is rotated into the site and placed between the covered tegmen and dural defects. The cranioplasty is performed by securing a titanium burr hole cover with 3-mm titanium screws (Fig. 1B). The remaining temporalis muscle is advanced and closed with interrupted dissolvable sutures. The temporalis muscle and skin are closed in layers with absorbable sutures. A layer of cyanoacrylate tissue adhesive (Dermabond, Ethicon Inc.) is used to cover the incision (Fig. 1C). No head dressing is necessary.

Patients are monitored in the neurosurgical intensive care unit for 2-3 days while the lumbar drain remains in place. They are typically discharged at postoperative Day 4 or 5 . Incisions are well healed 1 week after discharge (Fig. 1D).

\section{Statistical Analysis}

Analyses of data were performed using standard statistical methods, and data comparisons were calculated using the Student t-test on GraphPad Prism version 6 (GraphPad Software Inc.). Differences in data were considered significant at $\mathrm{p}<0.05$.

\section{Results}

Six procedures were performed in 5 patients (1 patient 

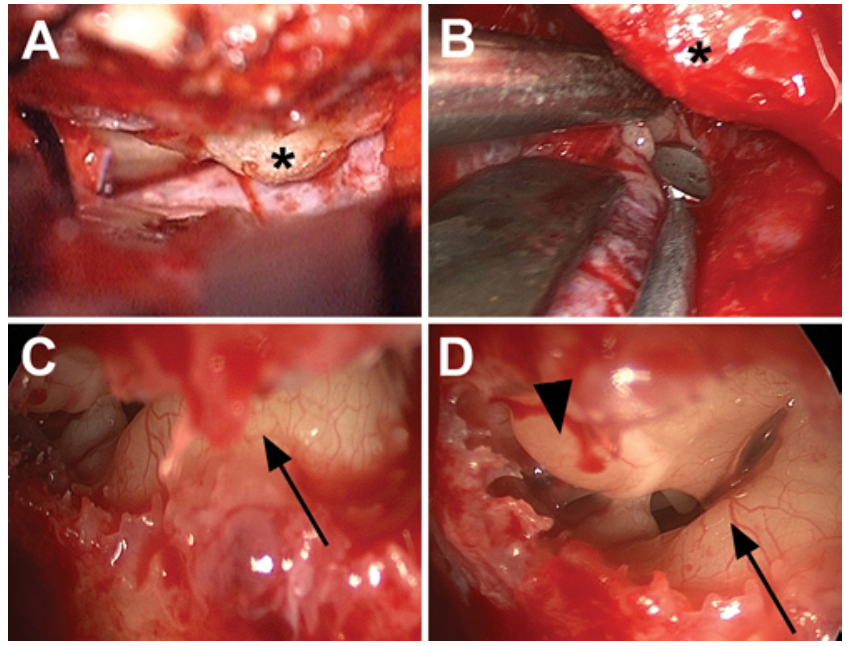

FIG. 2. Case 3. Visualization of the tegmen defect utilizing the operating microscope (A), $0^{\circ}$ endoscope (B), $30^{\circ}$ endoscope (C), and $70^{\circ}$ endoscope (D). Asterisks mark a shelf of bone overlying the tegmen dehiscence, arrows indicate bone of the middle ear cavity, and the arrowhead indicates the head of the malleus. Figure is available in color online only.

had bilateral TLEs) consisting of 4 women and 1 man. The average age at surgery was $57 \pm 11.7$ years (mean \pm SD). Four procedures were performed on the left ear and 2 on the right. Four of the procedures were performed in patients with chronic otitis media. In these 4 patients, the encephaloceles resulted from erosion of the bone due to repeated infections. The other 2 procedures were required for repair of spontaneously formed bilateral TLEs in a woman with a BMI of 41.5. The average BMI of the patients was $31.8 \pm 9.1$.

Hearing loss was a complaint of all patients (Table 1). Vertigo and imbalance were complaints of the majority of patients (4 ears belonging to 3 patients). Symptoms at presentation also included chronic tympanostomy tube otorrhea (4/6 ears) and chronic serous otitis media (2/6 ears).

The mean $( \pm$ SEM) opening CSF pressure was $18.7 \pm$ $3.5 \mathrm{~cm} \mathrm{H}_{2} \mathrm{O}$ in 3 of the 6 cases (CSF pressure was not measured in 2 patients). Half of the tegmen defects were larger than $1 \mathrm{~cm}$. The total time under anesthesia was 299 \pm 25 minutes (Table 2). The duration of the surgical procedures not including lumbar drain placement was $159.3 \pm$ 17 minutes, which included a mastoidectomy on a patient with suppurative otitis media. Tegmen repairs including opening and closure of skin averaged $144.5 \pm 23$ minutes.

Computed tomography scanning and intraoperative endoscopic photographs for Case 5 are shown in Fig. 3. Because endoscopes were used for visualization of the dural and tegmen defects, minimal retraction was required in all cases (Fig. 4). Postoperatively, the patients fared well. The average hospitalization was 5 days (range 4-6 days). A video of a typical endoscope-assisted repair is shown in the video supplement, as is a comparison video of a typical conventional middle fossa craniotomy and repair of a TLE (Videos 1 and 2, respectively).

VIDEO 1. Conventional middle fossa craniotomy with repair of

TLE and tegmen tympani dehiscence. Copyright Pamela Roehm.

Published with permission. Click here to view.
TABLE 1. Presenting symptoms in 5 patients who underwent endoscope-assisted repair of the tegmen mastoideum or tympani

\begin{tabular}{lc}
\hline \multicolumn{1}{c}{ Symptom } & No. of Ears $(\%)$ \\
\hline Hearing loss & $6(100)$ \\
\hline Otorrhea & $4(67)$ \\
\hline Vertigo & $4(67)$ \\
\hline Chronic serous otitis media & $2(33)$ \\
\hline
\end{tabular}

VIDEO 2. Endoscopic repair of TLE and tegmen tympani dehiscence via keyhole craniotomy. Copyright Pamela Roehm. Published with permission. Click here to view.

Patients were followed up for an average of 310 days (range 110-421 days). No major complications were noted. One patient developed a stitch abscess, which was treated with a short course of oral antibiotics. There were no recurrences of CSF otorrhea or encephaloceles.

Most patients experienced postoperative improvement in hearing, with the most significant improvements seen in patients with spontaneous CSF otorrhea and TLEs who had no history of underlying chronic otitis media. One patient failed to follow up for a postoperative audiogram. The remaining 4 patients had postoperative audiograms 2 months after surgery. The mean preoperative PTA was $48.75 \pm 6.0 \mathrm{~dB}$ sensorineural hearing loss (SNHL), which improved to $31.3 \pm 1.5 \mathrm{~dB}$ SNHL 2 months postoperatively $(\mathrm{p}=0.056)$. The mean air-bone gap improved from 26.4 \pm 7.7 preoperatively to $10.0 \pm 2.9(\mathrm{p}=0.08)$. Word recognition scores also improved from $93.3 \%$ preoperatively to $97.6 \%$ postoperatively, although this difference did not reach statistical significance in this small series $(p=0.15)$.

\section{Discussion}

Dehiscences in the tegmen tympani and/or mastoideum can occur from a number of causes, including infectious etiologies (acute or chronic otitis media and cholesteatoma), head trauma, iatrogenic injury, tumors (both intraaxial and extraaxial), osteoradionecrosis, and spontaneously or idiopathically. ${ }^{11}$ Spontaneous defects in the tegmen bone are associated with a high BMI $>25$ $\mathrm{kg} / \mathrm{m}^{2}$ ), benign intracranial hypertension, and obstructive sleep apnea and have increased in frequency concomi-

TABLE 2. Surgical and postoperative parameters in 5 patients who underwent endoscope-assisted repair of the tegmen mastoideum or tympani

\begin{tabular}{lc}
\hline \multicolumn{1}{c}{ Variable } & \multicolumn{1}{c}{ Value } \\
\hline CSF opening pressure in ${\mathrm{cm} \mathrm{H}_{2} \mathrm{O}^{*} \text { (SEM) }}^{\text {Average duration of anesthesia in mins (SEM) }}$ & $18.7(3.53)$ \\
\hline $\begin{array}{l}\text { Average duration of craniotomy and tegmen } \\
\text { repair in mins (SEM) }\end{array}$ & $144.5(25)$ \\
\hline Average length of stay in days (range) & $5(4-6)$ \\
\hline Average length of follow-up in days (range) & $310(110-421)$ \\
\hline$*$ Cerebrospinal fluid opening pressure measured in 3 of 5 patients.
\end{tabular}



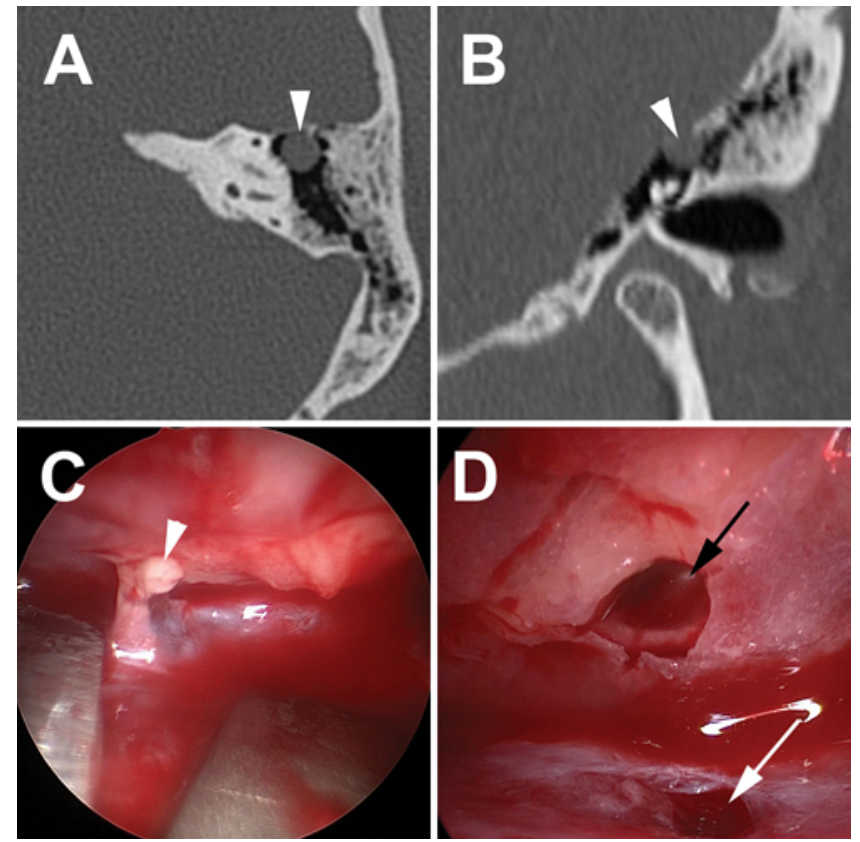

FIG. 3. Case 5. Axial CT showing encephalocele (arrowhead) in anterior epitympanum (A). Coronal CT demonstrating encephalocele (arrowhead) through a defect in the anterior epitympanum (B). Endoscopic visualization of encephalocele (arrowhead) using $0^{\circ}$ scope (C). Dural defect (white arrow) and tegmen defect (black arrow) demonstrated with $30^{\circ}$ endoscope (D). Figure is available in color online only.

tantly with the expansion of the obesity epidemic. ${ }^{4,10,14} \mathrm{~Pa}$ tients with tegmen dehiscences may present with hearing loss due to chronic serous otitis media or direct contact of TLEs onto the ossicles, chronic tube otorrhea, pulsatile tinnitus, and imbalance. Appropriate evaluation of suspected CSF otorrhea or TLEs includes complete audiography with tympanometry, CT of the temporal bones to detect tegmen dehiscences, and MRI of the internal auditory canals and brain to definitively identify encephaloceles. ${ }^{11}$

Appropriate management of TLEs is surgical repair. Classically, these defects have been approached via mastoidectomy or middle fossa craniotomy or a combination of the two, with microscopic visualization of the defect. ${ }^{5,8,11,14}$ All of these approaches have limitations. For instance, not all tegmen defects can be visualized through the transmastoid approach. Visualization through the mastoidectomy cavity is limited to defects of the tegmen mastoideum lateral to the superior semicircular canal. Successful closure of tegmen defects medial to the semicircular canal cannot be achieved through a mastoidectomy. Defects in the tegmen overlying the epitympanum and anterior to that area cannot be approached through a mastoidectomy. Attempted closure in these areas endangers hearing when the ossicular chain is intact. Visualization of tegmen defects from this approach is also poor in these areas unless the posterior canal wall is taken down. Repair of large defects from below (through a mastoid approach) can also be difficult. A middle fossa approach allows visualization of the entire tegmen without risk to the ossicular chain. However, defects in the posterior mastoid cavity_for instance,
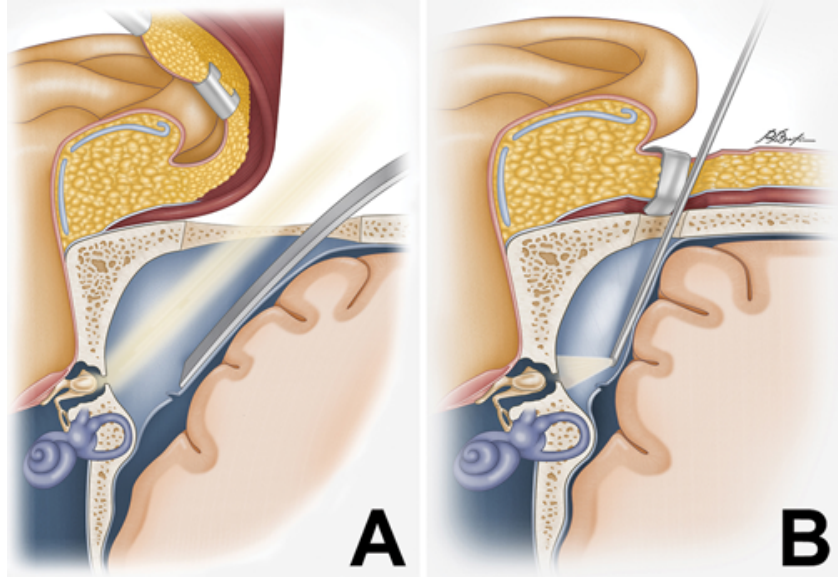

FIG. 4. Use of a microscope through a middle fossa craniotomy requires extradural retraction to allow full visualization of tegmen defects, particularly medial defects (A). Visualization through an angled endoscope allows visualization of skull base defects with significantly less retraction (B). Copyright Pamela Roehm. Published with permission. Figure is available in color online only.

through the cerebellar plate-cannot be repaired through a middle cranial fossa approach.

Extradural retraction of the brain is required for visualization of tegmen defects through a middle fossa approach. Brain retraction is associated with a number of adverse effects on the brain, including cerebral edema, decreases in blood flow, ischemia, and partial loss of the blood-brain barrier. ${ }^{1-3,7,12,13}$ Retraction of the brain results in changes in the levels of a variety of proteins, which can affect neuronal survival and cerebral blood flow., ${ }^{9,13}$ Although the dura partially protects the brain during retraction and spreads the pressure of retraction over a wider area, pressures of up to $8.2 \mathrm{~mm} \mathrm{Hg}$ have been measured while using standard middle fossa retractors to visualize the lateral skull base. ${ }^{6}$ Thus, avoiding retraction is desirable in middle fossa surgery.

In this paper we present a series of 6 cases in 5 patients with tegmen dehiscences. For all of these patients, we used an endoscope in addition to the binocular microscope for visualization. The nasal endoscope was used to fully visualize the tegmen defect, which allowed us to perform these surgeries through a keyhole craniotomy. We were also able to use a far smaller incision for the procedure than previously required with standard visualization using binocular microscopy via a middle cranial fossa approach. The use of endoscopes for visualization allowed us to perform the surgery with minimal middle fossa retraction. When TLEs were present, they were reduced without difficulty using endoscopic visualization. In our series of 6 procedures, there were no major complications. The only minor complication was a suture abscess in 1 patient that resolved with topical therapy and oral antibiotics. Length of stay, duration of CSF drainage, and duration of procedure were not significantly affected by the additional use of endoscopy for visualization during these procedures.

While we have not experienced any limitations of this procedure to date, ultimately limitations will occur. Potentially, visualization and repair of very large defects could 
be difficult, particularly for a procedure that is visualized completely via endoscopy. The small size of the craniotomy limits the amount of bone available for tegmen repair, and for larger defects, other sources of bone may be required. Finally, there is a learning curve for endoscopic procedures, and for surgeons unfamiliar or lacking recent experience with endoscopy, longer operating times could be anticipated early during mastery of this technique.

This study used endoscopic techniques to repair defects of the lateral skull base and temporal bones, which allowed the use of much smaller (keyhole) craniotomies and smaller skin incisions to perform repairs of skull base bony defects and TLEs. Other approaches are available, including conventional middle fossa craniotomy, transmastoid approaches, and combinations of conventional middle fossa and transmastoid approaches. Limitations of this study include the relatively small number of patients and the relatively limited duration of follow-up.

\section{Conclusions}

Middle fossa tegmen defects and TLEs can be repaired via middle fossa craniotomy, transmastoid approach, or combined approaches, based on the position of the defect. When a conventional middle fossa craniotomy technique is used, dural retraction must be performed to fully visualize the defect and allow for repair. In this study, we sought to determine if the use of endoscopic techniques could provide sufficient visualization to identify and repair these defects. We found that endoscopic visualization not only allowed us to successfully repair these defects, but also enabled us to use a much smaller incision and substantially decrease the amount of retraction required.

\section{Acknowledgments}

This study was financially supported by National Institutes of Health, National Institute on Deafness and Other Communication Disorders (NIH/NIDCD) Grant No. K08DC009288 (P.C.R.).

\section{References}

1. Albin MS, Bunegin L, Helsel P, Marlin A, Babinski M: Intracranial pressure and regional cerebral blood flow responses to experimental brain retraction pressure, in Shulman K, Marmarou A, Miller J, et al (eds): Intracranial Pressure IV. Berlin: Springer, 1980, pp 131-135

2. Andrews RJ, Bringas JR: A review of brain retraction and recommendations for minimizing intraoperative brain injury. Neurosurgery 33:1052-1064, 1993

3. Andrews RJ, Muto RP: Retraction brain ischaemia: cerebral blood flow, evoked potentials, hypotension and hyperventilation in a new animal model. Neurol Res 14:12-18, 1992

4. Bakhsheshian J, Hwang MS, Friedman M: Association between obstructive sleep apnea and spontaneous cerebrospinal fluid leaks: a systematic review and meta-analysis. JAMA Otolaryngol Head Neck Surg 141:733-738, 2015

5. Brown NE, Grundfast KM, Jabre A, Megerian CA, O'Malley BW Jr, Rosenberg SI: Diagnosis and management of spontaneous cerebrospinal fluid-middle ear effusion and otorrhea. Laryngoscope 114:800-805, 2004

6. Driscoll CL, Jackler RK, Pitts LH, Banthia V: Extradural temporal lobe retraction in the middle fossa approach to the internal auditory canal: biomechanical analysis. Am J Otol 20:373-380, 1999

7. Fukamachi A, Koizumi H, Nukui H: Postoperative intracerebral hemorrhages: a survey of computed tomographic findings after 1074 intracranial operations. Surg Neurol 23:575-580, 1985

8. Kenning TJ, Willcox TO, Artz GJ, Schiffmacher P, Farrell CJ, Evans JJ: Surgical management of temporal meningoencephaloceles, cerebrospinal fluid leaks, and intracranial hypertension: treatment paradigm and outcomes. Neurosurg Focus 32(6):E6, 2012

9. Nag S, Manias JL, Stewart DJ: Pathology and new players in the pathogenesis of brain edema. Acta Neuropathol 118:197-217, 2009

10. Nelson RF, Gantz BJ, Hansen MR: The rising incidence of spontaneous cerebrospinal fluid leaks in the United States and the association with obesity and obstructive sleep apnea. Otol Neurotol 36:476-480, 2015

11. Raine C: Diagnosis and management of otologic cerebrospinal fluid leak. Otolaryngol Clin North Am 38:583-595, 2005

12. Rosenørn J, Diemer NH: Reduction of regional cerebral blood flow during brain retraction pressure in the rat. J Neurosurg 56:826-829, 1982

13. Sherchan P, Kim CH, Zhang JH: Surgical brain injury and edema prevention. Acta Neurochir Suppl 118:129-133, 2013

14. Vivas EX, Mccall A, Raz Y, Fernandez-Miranda JC, Gardner P, Hirsch BE: ICP, BMI, surgical repair, and CSF diversion in patients presenting with spontaneous CSF otorrhea. Otol Neurotol 35:344-347, 2014

\section{Disclosures}

The authors report no conflict of interest concerning the materials or methods used in this study or the findings specified in this paper.

\section{Author Contributions}

Conception and design: Roehm, Erkmen. Acquisition of data: Roehm, Tint, Chan, Sukul, Erkmen. Analysis and interpretation of data: Roehm, Chan, Sukul, Erkmen. Drafting the article: all authors. Critically revising the article: Roehm, Sukul, Erkmen. Reviewed submitted version of manuscript: Roehm, Erkmen. Approved the final version of the manuscript on behalf of all authors: Roehm. Statistical analysis: Roehm, Chan. Administrative/technical/material support: Tint, Chan, Brewster. Study supervision: Roehm, Erkmen.

\section{Supplemental Information Videos \\ Video 1. https://vimeo.com/208664682. \\ Video 2. https://vimeo.com/208664827.}

\section{Previous Presentations}

This work was previously presented at the American Neurotology Society Meeting held in Chicago, Illinois, in May 2016.

\section{Correspondence}

Pamela C. Roehm, Department of Otolaryngology, Temple University School of Medicine, 3509 N Broad St., 6th Fl. Boyer Pavilion, Philadelphia, PA 19140. email: pamela.roehm@tuhs. temple.edu. 